\title{
Hemopoietic Colony Growth-promoting Activities In the Plasma of Bone Marrow Transplant Recipients
}

\author{
Kazuo Yamasaki, Lawrence A. Solberg, Jr.," Nazir Jamal, Gina Lockwood, David Tritchler, John E. Curtis, \\ Mark M. Minden, Kenneth G. Mann, ${ }^{\ddagger}$ and Hans A. Messner \\ Ontario Cancer Institute, Institute of Medical Science, University of Toronto, Canada, M4X 1K9; *Mayo Clinic,

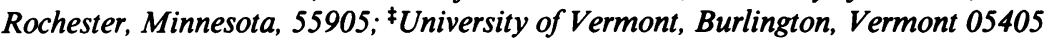

\begin{abstract}
Plasma samples were obtained from 34 bone marrow transplant (BMT) recipients before and after administration of the preparative regimen and tested for their ability to promote and/or support growth of hemopoietic colonies. The ability of plasma samples to promote colony formation on their own was tested on normal nonadherent target cells without addition of exogenous growth factors. The growth-supporting activity was examined in the presence of medium conditioned by phytohemagglutinin-stimulated leukocytes (PHA-LCM) and/or erythropoietin (EPO). A series of kinetic changes was routinely observed. Pretransplant samples rarely gave rise to colonies without addition of exogenous growth factors. Plasma samples obtained after completion of the preparative regimen demonstrated increments of growth-promoting activities for megakaryocyte and granulocyte-macrophage progenitors (CFUMeg and CFU-GM), respectively, that peaked between 7 and 21 d after transplantation. By day 30, activity levels of some patients had returned to pretransplant values, whereas in other patients, activities remained elevated. Persisting activity levels were associated with delayed engraftment. In contrast, activities for progenitors committed to erythropoiesis (BFU-E) and pluripotent precursors (CFU-GEMM) were only rarely observed. The activities were independent of febrile episodes. Their growth-promoting influence on CFU-GM could be neutralized completely by anti-granulocyte-macrophage colonystimulating factor (GM-CSF) antibodies. These data suggest that at least some of the observed activities in post-BMT plasma are related to GM-CSF. The growth-supporting activities of pretransplant plasma samples are lower than normal plasma when tested on CFU-Meg and CFU-GM. The growthsupporting activities improved transiently within the first month after BMT. A decline during the second and third month was followed by a gradual return to activity levels that were comparable to normal plasma. The effects of these
\end{abstract}

Portions of this work were presented at the 1983 and 1986 meetings of the American Society of Hematology, and were published in abstract form (1983. Blood. 62[Suppl. 1]:147a and 1986. Blood. 68[Suppl. 1]:284a).

Address correspondence to Dr. Messner, Ontario Cancer Institute, 500 Sherbourne Street, Toronto, Ontario, Canada, M4X 1 K9.

Received for publication 31 July 1987 and in revised form 21 December 1987.

J. Clin. Invest.

(C) The American Society for Clinical Investigation, Inc.

0021-9738/88/07/0255/07 $\$ 2.00$

Volume 82, July 1988, 255-261 plasma samples on BFU-E and CFU-GEMM were assessed with PHA-LCM and EPO. Similar to CFU-Meg- and CFUGM-supporting capabilities, they improved transiently after BMT with a return of normal support function after 5-6 mo. The observed endogenous production of growth-promoting and growth-supporting activities for hemopoietic progenitors may serve as a background to design clinical trials for the timely administration of recombinant hemopoietic growth factors to BMT recipients.

\section{Introduction}

Human recombinant hemopoietic growth factors are now available and have shown effectiveness in cell culture (1-10). Preclinical and clinical studies have also demonstrated their ability to increase the level of peripheral blood cells in vivo (11-15). A well-timed administration of these growth factors to bone marrow transplant (BMT) ${ }^{1}$ recipients may provide the opportunity to improve the speed and quality of bone marrow recovery after BMT (16). In a previous study (17) now confirmed (18), we were able to demonstrate that plasma samples collected from $11 \mathrm{BMT}$ recipients contained activities that promoted growth of progenitors committed to megakaryocytopoiesis (CFU-Meg) and granulocyte-macrophage production (CFU-GM). Some of these samples also facilitated the development of erythroid bursts, while multilineage colonies were not observed. Operationally, the described activities supported and promoted the growth of hemopoietic progenitors replacing the requirement for an additional source of exogenous growth factors completely or at least partially. This contrasts with the finding that normal plasma samples give rise to hemopoietic colonies only if a source of exogenous growth factors is added to the cultures (19). It is the purpose of this investigation to describe growth-supporting and growth-promoting, growth factor-like activities in the plasma of BMT recipients collected before and serially after BMT. In addition, attempts are made to determine whether or not a correlation exists between the kinetic behavior of these activities and engraftment.

1. Abbreviations used in this paper: ALL, acute lymphoblastic leukemia; AML, acute myeloid leukemia; BFU-E, progenitor committed to erythropoiesis; BMT, bone marrow transplantation; CFU-GEMM, pluripotent precursor; CFU-GM, progenitor committed to granulocyte-macrophage production; CFU-Meg, progenitor committed to megakaryocytopoiesis; CML, chronic myeloid leukemia; EPO, erythropoietin; GM-CSF, granulocyte-macrophage colony-stimulating factor; GvHD, graft vs. host disease; IL3, Interleukin 3 2ME, 2-mercaptoethanol; PHA-LCM, medium conditioned by PHA-stimulated leukocytes; rg, recombinant gibbon; rh, recombinant human; WRS, Wilcoxon rank-sum test; WSR, Wilcoxon signed rank test. 


\section{Methods}

Patients. 34 consenting patients that received marrow grafts from HLA-identical sibling donors were studied prospectively. These included eight individuals with acute myeloid leukemia (AML), seven with acute lymphoblastic leukemia (ALL), 14 with chronic myeloid leukemia (CML), one with aplastic anemia, three with multiple myeloma, and 1 with malignant lymphoma. In addition, plasma samples of 27 additional patients were examined who had at least survived $1 \mathrm{yr}$ after BMT. All recipients were prepared according to previously published protocols (20). Graft versus host disease (GvHD) prophylaxis included administration of four doses of methotrexate followed by prednisone $40 \mathrm{mg} / \mathrm{m}^{2}$ per $\mathrm{d}$ from day 10 to 40 with slow dose reduction over the subsequent $100 \mathrm{~d}$. Patients who developed acute GvHD of grades II-IV were treated with cyclosporin A either orally at $12.5 \mathrm{mg} / \mathrm{d}$ or intravenously at $5 \mathrm{mg} / \mathrm{d}(21)$.

Preparation of plasma samples. A total of 247 heparinized plasma specimens were collected from BMT recipients before administration of the conditioning regimen ( 32 samples) and serially after transplantation (215 samples). The samples were obtained fasting and at least 12-24 $\mathrm{h}$ after the last transfusion of blood products. They were prepared under platelet poor conditions as previously described (17) and stored frozen at $-20^{\circ} \mathrm{C}$ until testing. The tests were performed within 6 mo of collection.

Preparation of target cells. Heparinized bone marrow samples were obtained from consenting normal bone marrow transplant donors. Mononuclear cells of density $<1.077 \mathrm{~g} / \mathrm{ml}$ were prepared by centrifugation in Percoll (Pharmacia Fine Chemicals, Uppsala, Sweden) and extensively depleted of adherent cells $(17,19,22)$.

In some experiments, nonadherent mononuclear target cells were further depleted of $\mathrm{T}$ lymphocytes using a double rosetting procedure with 2-aminoethylisothio-uronium bromide hydrobromide treated sheep red blood cells (23).

Bioassay for hemopoietic growth factors in human plasma. Nonadherent bone marrow cells at $2 \times 10^{5} / \mathrm{ml}$ were routinely cultured $(17$, 22 ) in Iscove's modification of Dulbecco's medium (Gibco, Grand Island, NY) supplemented with $5 \times 10^{-5} \mathrm{M}$ 2-mercaptoethanol (2ME) (Sigma Chemical Co., St. Louis, MO), 0.9\% methylcellulose (Dow Chemical Company, Midland, MI), 30\% normal human plasma, 10\% medium conditioned by phytohemagglutinin-stimulated leukocytes (PHA-LCM, PHA, HA 15, Wellcome Diagnostic, Dartford, England) (24), and 1 U of human urinary erythropoietin (EPO, Terry Fox Laboratory, Vancouver, $\mathrm{BC}$ ). The use of human plasma at a concentration of $30 \%$ was previously determined as supporting optimal colony formation $(19,22)$. Colonies were scored after $14 \mathrm{~d}$ of culture in a humidified atmosphere containing $5 \% \mathrm{CO}_{2}$ using previously described criteria $(19,22,23,25)$. In addition, each nonadherent target cell population was also grown under the following three conditions: with normal human plasma, without further sources of growth factors; with normal human plasma and EPO; and with normal human plasma and PHA-LCM.

Cultures containing only normal human plasma were evaluated to determine the formation of background colonies by the nonadherent target cell population in the absence of exogenous growth factors. Cultures with normal plasma and PHA-LCM were considered to represent maximal formation of megakaryocyte and granulocyte-macrophage colonies. Cultures containing normal plasma, PHA-LCM, and EPO gave rise to maximal colony formation by pluripotent precursors (CFU-GEMM) and progenitors committed to erythropoiesis (BFU-E).

All plasma samples obtained from BMT recipients were tested at a concentration of $30 \%$ under the same four conditions as above by replacing normal human plasma. The frequency of colonies was recorded and the results were expressed as a percentage of colony values determined in the respective control groups. Background colony formation with normal human plasma alone was taken into account. All serially collected plasma samples from each BMT recipient were tested on the same set of nonadherent target cells.
Neutralization studies with anti-GM-CSF and anti-IL-3. Recombinant human granulocyte-macrophage colony-stimulating factor (rh GM-CSF) $(1,26)$ and recombinant gibbon ( $\mathrm{rg} 9,26)$ IL-3 were obtained as supernantants of chinese hamster ovary cells and monkey COS- 1 cells which had been transfected with the respective genes. Both growth factors as well as affinity-purified antibody preparations (9) raised in sheep against rh GM-CSF and in rabbits against rg IL-3 were a generous gift of Dr. S. C. Clark (Genetics Institute, Boston, MA).

$150 \mu$ l of each plasma sample obtained from BMT recipients were incubated in Eppendorff tubes for $1 \mathrm{~h}$ at $37^{\circ} \mathrm{C}$ using the antibody preparations in dilutions from 1:50 to 1:5,000 and kept overnight at $4^{\circ} \mathrm{C}$. The resulting immune complexes were removed from the plasma by centrifugation at $1,500 \mathrm{~g}$ after a $2-\mathrm{h}$ incubation at $4^{\circ} \mathrm{C}$ with protein G-bearing Omnisorb cells (Calbiochem, Terochem Laboratories, Mississauga, Ontario) (27). The plasma specimens were tested without further addition of growth factors at a final concentration of $30 \%$ using $5 \times 10^{3}$ E rosette-depleted (23), nonadherent normal bone marrow cells plated in flat-bottomed microtiter plates (Nunclon, Gibco Canada, Burlington, Ontario), in a volume of $0.1 \mathrm{ml}$ containing methylcellulose, 2ME, and Iscove's modified Dulbecco's medium as described above. The target cell population was also plated with rh GMCSF and rg IL-3 as well as growth factor preparations that had been preincubated with anti-GM-CSF and anti-IL-3.

Statistical methods. The activity levels used in the statistical analysis were calculated as a percentage of the maximal colony formation of normal controls above the background colony formation.

For the purpose of this study, time to engraftment was defined for each lineage as the time elapsing between the transplant and the day when $>50,000$ platelets, $>50,000$ reticulocytes, or $>1,500$ granulocytes $/ \mathrm{mm}^{3}$ of peripheral blood were recorded and subsequently sustained without transfusions. Platelet counts on the day of or on the day after platelet transfusions were not considered for this study. None of the patients received granulocyte transfusions. Each patient was evaluated for the presence of GvHD following established criteria (28). The Wilcoxon rank-sum test (WRS) was used for comparisons between groups of measurements (29). The Wilcoxon signed rank test (WSR) was used to test the location of the mean of a population of measurements (29).

\section{Results}

All samples were tested on normal nonadherent target cells for growth-promoting activities in the absence of other exogenous sources of growth factors. In addition, specimens were examined for their ability to support growth of hemopoietic progenitors in the presence of PHA-LCM. The majority of target cells did not form colonies when plated with normal human plasma in the absence of additional growth factors.

\section{Activities for CFU-Meg}

Testing of plasma samples without PHA-LCM. Plasma samples collected from BMT recipients before transplantation usually contained activities that promoted growth of a small number of megakaryocyte colonies in the absence of PHALCM (Fig. 1). The activity levels increased by the day of the transplant (day 0 ), determined before infusion of the bone marrow. Maximal levels were reached between days 7 and 26. These varied considerably from patient to patient. Plasma of some patients promoted growth of more megakaryocyte colonies than normal human plasma and PHA-LCM, while samples obtained from other patients resulted in suboptimal colony formation.

The kinetic activity patterns of individual patients demonstrated considerable heterogeneity. Three examples of patients 


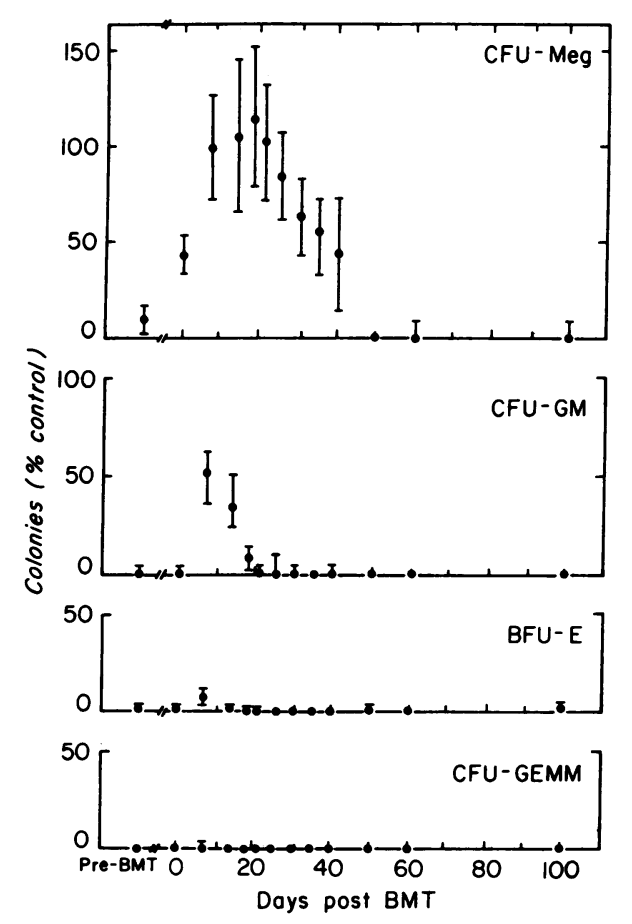

Figure 1. Colony formation by normal nonadherent target cells plated with plasma obtained from bone marrow transplant recipients before and serially after BMT. The cultures did not contain any other exogenous source of growth factors. The data are expressed as percent of normal controls. Controls for CFU-Meg and CFU-GM were plated with $30 \%$ normal human plasma and 10\% PHA-LCM. Controls for BFU-E and CFU-GEMM contained also $1 \mathrm{U}$ of EPO. The following control values (mean \pm SEM) per $2 \times 10^{5}$ mononuclear cells were observed for: CFU-Meg, $63 \pm 12$; CFU-GM, 189 \pm 24 ; BFU-E, 217 \pm 22 ; CFU-GEMM, $25 \pm 3$.

that had at least eight serial determinations are displayed in Fig. 2. A single activity peak with return to pretransplant values by day 28 (Fig. $2 \mathrm{~A}$ ) was observed in 15 patients (four ALL, four AML, four CML, two multiple myeloma, one malignant lymphoma). The activity levels in 13 patients (two ALL, three AML, seven CML, one multiple myeloma) were sustained beyond day 30 (Fig. $2 \mathrm{~B}$ ). The only patient in this study transplanted for aplastic anemia showed a different activity profile (Fig. $2 \mathrm{C}$ ). The pretransplant plasma promoted megakaryocyte colony growth better than controls plated with normal plasma and PHA-LCM. The activity declined gradually and by day 60 the plasma behaved like normal plasma.

The data points collected for five patients were not sufficient to assign them to a specific activity profile. All available plasma samples of one of these five patients were void of stimulatory activity (Fig. $2 \mathrm{D}$ ). This patient suffered from CML complicated by severe myelofibrosis.

Plasma samples of four patients collected around day 14 after BMT were plated with $1 \times 10^{5}$ normal nonadherent target cells that were also extensively depleted of $T$ lymphocytes. This experiment was performed to determine whether or not the activities detected in plasma of BMT recipients require the presence of $\mathrm{T}$ lymphocytes. The pattern of megakarycyte colony formation by these $T$ cell-depleted target cells was similar to that obtained with nonadherent target cells that contained $T$ lymphocytes (data not shown).
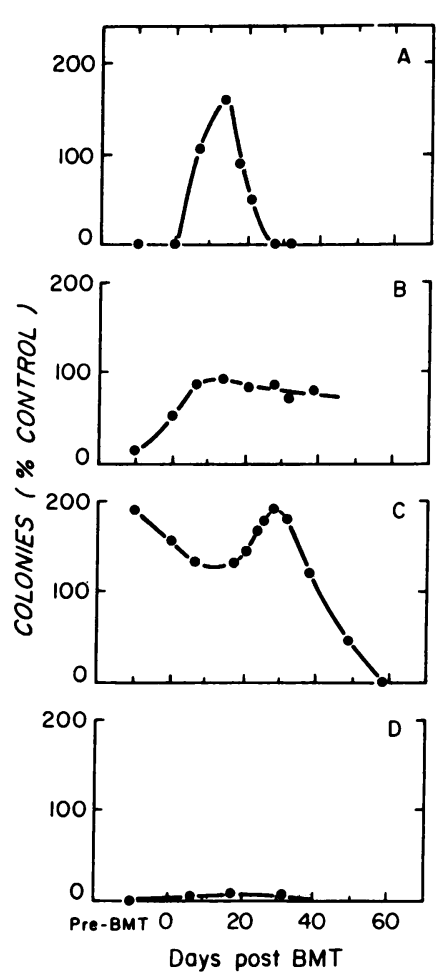

Figure 2. Megakaryocyte colony formation by normal nonadherent target cells using plasma samples of four different BMT recipients. All cultures were plated without further growth factors. Profiles $A$ and $B$ were most commonly observed in patients with various hemopoietic malignancies. The activity profile in $C$ was obtained for a patient with severe aplastic anemia. Profile $D$ shows the absence of any activity in a patient transplanted for CML with severe myelofibrosis.

Testing of plasma samples with PHA-LCM. The plasma samples were also evaluated in the presence of PHA-LCM (Table I). The frequency of colonies observed with plasma obtained before administration of the preparative regimen was significantly lower than with normal plasma. The plating efficiency increased with plasma obtained between days 0 and 30 of the BMT. The growth support exerted by plasma collected from BMT recipients between days 7 and 30 approximated the activity level usually observed with normal plasma. Plasma samples obtained between days 50 and 100 after BMT supported megakaryocyte colony growth in the presence of PHALCM to a significantly lesser degree than normal plasma: this was significant at the 0.05 level for days 50 and 100 (WSR, $P$ $=0.001$ and $P=0.030$, respectively) and significant at the 0.10 level for day 60 (WSR, $P=0.059$ ). Specimens studied 5-48 mo after BMT provided nearly normal growth support (Table I).

\section{Activities for CFU-GM}

Plasma samples obtained from BMT recipients before transplantation occasionally promoted growth of a small number of granulocyte-macrophage colonies in the absence of PHALCM (Fig. 1). Growth-promoting activities were routinely observed 7-14 d after BMT. The colony frequency approximated $50 \%$ of controls grown with normal plasma and PHA-LCM. The variability of activity profiles for individual patients was less compared to activities for CFU-Meg.

The frequency of granulocyte-macrophage colonies observed in the presence of PHA-LCM and plasma collected from BMT recipients before and during the first $30 \mathrm{~d}$ after BMT ranged from 69 to $94 \%$ of maximally stimulated controls (Table I). In addition, PHA-LCM also increased the size of individual colonies. Consistent with data for CFU-Meg, plasma samples obtained $\mathbf{4 0} \mathrm{d}$ or later after BMT supported 


\begin{tabular}{|c|c|c|c|c|c|c|c|}
\hline \multirow[b]{3}{*}{ Time } & \multirow[b]{3}{*}{$n^{*}$} & \multicolumn{6}{|c|}{ Colonies ( $\%$ control) } \\
\hline & & \multirow[b]{2}{*}{ CFU-Meg $1^{*}$} & \multirow[b]{2}{*}{ CFU-GM 1} & \multicolumn{2}{|c|}{ BFU-E } & \multicolumn{2}{|c|}{ CFU-GEMM } \\
\hline & & & & 1 & $2^{8}$ & 1 & 2 \\
\hline Pre BMT & 30 & $49 \pm 7^{11}$ & $74 \pm 10$ & $6 \pm 3$ & $84 \pm 8$ & $5 \pm 33$ & $87 \pm 10$ \\
\hline \multicolumn{8}{|c|}{ Post BMT $(d)$} \\
\hline 0 & 26 & $61 \pm 9$ & $81 \pm 16$ & $13 \pm 4$ & $58 \pm 20$ & $21 \pm 8$ & $53 \pm 29$ \\
\hline 7 & 29 & $76 \pm 12$ & $69 \pm 12$ & $28 \pm 7$ & $84 \pm 9$ & $16 \pm 5$ & $88 \pm 10$ \\
\hline 14 & 20 & $72 \pm 14$ & $94 \pm 18$ & $23 \pm 18$ & $73 \pm 16$ & $21 \pm 7$ & $71 \pm 20$ \\
\hline 21 & 25 & $84 \pm 11$ & $71 \pm 12$ & $14 \pm 6$ & $53 \pm 13$ & $11 \pm 6$ & $59 \pm 19$ \\
\hline 30 & 23 & $80 \pm 13$ & $69 \pm 15$ & $9 \pm 4$ & $54 \pm 12$ & $12 \pm 7$ & $81 \pm 16$ \\
\hline 40 & 14 & $60 \pm 22$ & $39 \pm 17$ & $1 \pm 1$ & $56 \pm 28$ & $2 \pm 2$ & $64 \pm 32$ \\
\hline 50 & 15 & $31 \pm 9$ & $36 \pm 17$ & $4 \pm 3$ & $19 \pm 13$ & $2 \pm 2$ & $12 \pm 8$ \\
\hline 60 & 6 & $21 \pm 18$ & $46 \pm 23$ & $0 \pm 0$ & $63 \pm 32$ & $1 \pm 1$ & $63 \pm 32$ \\
\hline 100 & 8 & $52 \pm 15$ & $44 \pm 16$ & $0 \pm 0$ & $35 \pm 12$ & $0 \pm 0$ & $66 \pm 23$ \\
\hline \multicolumn{8}{|c|}{ Post BMT (mo) } \\
\hline $5-12$ & 10 & $92 \pm 10$ & $84 \pm 7$ & $5 \pm 5$ & $76 \pm 9$ & $3 \pm 3$ & $95 \pm 11$ \\
\hline $13-24$ & 7 & $102 \pm 6$ & $90 \pm 5$ & $0 \pm 0$ & $86 \pm 4$ & $0 \pm 0$ & $103 \pm 4$ \\
\hline $25-36$ & 7 & $83 \pm 11$ & $90 \pm 7$ & $0 \pm 0$ & $77 \pm 10$ & $0 \pm 0$ & $75 \pm 18$ \\
\hline $37-48$ & 3 & $82 \pm 16$ & $90 \pm 8$ & $0 \pm 0$ & $86 \pm 10$ & $0 \pm 0$ & $93 \pm 2$ \\
\hline
\end{tabular}

* Number of recipients tested. ${ }^{\ddagger}$ Plated with PHA-LCM. ${ }^{\S}$ Plated with PHA-LCM and EPO. " Mean \pm SEM.

the growth of fewer granulocyte-macrophage colonies than normal plasma when tested with PHA-LCM. Days 40 and 50 were significantly different from normal at the 0.05 level (WSR, $P=0.003$ and 0.030 ). The activity on day 100 differed at the 0.10 level (WSR, $P=0.093$ ). A return to normal supporting activities was observed 5 mo after BMT (Table I).

\section{Activities for BFU-E and CFU-GEMM}

Erythroid bursts and multilineage colonies with erythroid components were only occasionally observed when normal nonadherent target cells were plated with plasma in the absence of PHA-LCM and exogenous EPO. Addition of EPO to the cultures did not improve the plating efficiency significantly. The use of PHA-LCM without exogenous EPO resulted in the formation of some erythroid bursts and multilineage colonies when plasma samples obtained from recipients early after BMT were examined. The plating efficiency of BFU-E and CFU-GEMM, however, did not exceed $30 \%$ of the established maximal controls. This observation suggests that plasma samples collected within the first 4-6 wk after BMT contain some EPO. The plating efficiency was further improved when EPO and PHA-LCM was added to the cultures. Plasma samples collected around day 50 again demonstrated a deficiency in supporting growth of BFU-E and CFU-GEMM under otherwise maximally stimulated culture conditions.

Long-term observation indicated that the growth-supporting activity improved in plasma samples collected 5 mo or later after BMT (Table I).

Neutralization of growth-promoting activities for CFUGM in post-BMT plasma by anti-GM-CSF antibodies

Post-BMT plasma samples were exposed to anti-GM-CSF and anti-IL-3 antibodies to determine whether or not the activities identified in the biological assay system were related to known hemopoietic growth factors. The E rosette-depleted, nonadherent target cells did not form colonies in the absence of growth factors, but gave rise to granulocyte-macrophage colonies when either rh GM-CSF or rg IL-3 were added (Table II). Colony formation under the influence of rh GM-CSF and $\mathrm{rg}$ IL-3 was abrogated by preincubation with respective antibody preparations. Similarly, the growth promoting activity for CFU-GM identified by bioassay in six tested plasma samples was completely neutralized after preincubation with antiGM-CSF. The neutralization was concentration dependent (Table II). In contrast, similar studies with anti-IL-3 on two plasma samples did not result in a reduction of the growth promoting activity.

The frequency of megakaryocyte colonies per microwell was too low to permit definitive conclusions about the influence of anti-GM-CSF and anti-IL-3 on plasma activities that promote the growth of megakaryocyte colonies.

\section{Clinical correlation}

Influence of plasma activities on time to engraftment. We compared the growth-promoting activities in plasma samples before and after BMT with time to recovery of single lineage peripheral blood counts. The distributions of the plasma activities at each time were determined. The times to recovery of platelets, granulocytes, and reticulocytes were compared for patients with activities above and below the median. Only the day 30 post-BMT activity level for megakaryocyte precursors was significantly related to the time to engraftment as determined for platelets. Patients with activity levels less than the median tended to engraft faster than patients with activity levels greater than the median (WRS, $P=0.017$ ). A similar trend was observed for stimulating activities for granulocytemacrophage precursors. Higher activities than the median, around day 30, were associated with slower engraftment as 
Table II. Influence of Anti-GM-CSF and Anti-IL-3 on Stimulating Activities for CFU-GM in Post-BMT Plasma

\begin{tabular}{|c|c|c|c|c|c|c|c|}
\hline \multirow[b]{2}{*}{ Source of plasma } & \multirow[b]{2}{*}{ Growth factors } & \multirow[b]{2}{*}{ Preparations } & \multicolumn{5}{|c|}{ No. of GM colonies* with different antibody concentrations } \\
\hline & & & 0 & $1: 5,000$ & $1: 1,000$ & $1: 100$ & 1:50 \\
\hline Normal & 0 & 0 & 0 & ND & ND & ND & ND \\
\hline Normal & GM-CSF ${ }^{\ddagger}$ & Anti-GM-CSF & 15 & 12 & 1 & 0 & ND \\
\hline Normal & IL-3 $3^{\S}$ & Anti-IL-3 & 6 & 5 & 0 & 0 & ND \\
\hline BMT $140(11)^{\|}$ & 0 & Anti-GM-CSF & 19 & 8 & 11 & 2 & ND \\
\hline BMT $156(4)$ & 0 & Anti-GM-CSF & 5 & 1 & 0 & 0 & ND \\
\hline BMT $156(15)$ & 0 & Anti-GM-CSF & 9 & 2 & 1 & 0 & ND \\
\hline BMT 200 (7) & 0 & Anti-GM-CSF & 18 & ND & 10 & 4 & 0 \\
\hline BMT 200 (14) & 0 & Anti-GM-CSF & 14 & ND & 5 & 0 & 0 \\
\hline BMT 210 (7) & 0 & Anti-GM-CSF & 17 & 16 & 13 & 0 & 0 \\
\hline BMT 200 (7) & 0 & Anti-IL-3 & 18 & ND & 20 & 19 & 16 \\
\hline BMT 200 (14) & 0 & Anti-IL-3 & 14 & ND & 12 & 13 & 14 \\
\hline
\end{tabular}

ND, not done. ${ }^{*} 5 \times 10^{3} \mathrm{E}$ rosette-depleted nonadherent cells plated in microwells. ${ }^{\ddagger} \mathrm{GM}$-CSF 1:104 dilution of Chinese hamster ovary cell supernatant. ${ }^{\S} \mathrm{IL}-3,1: 5,000$ dilution of COS-1 cell supernatant. "Days after BMT, when plasma was collected.

determined for granulocytes, although the difference was not significant at the 0.05 level (WRS, $P=0.059$ ). Activities higher than the median for BFU-E observed at day 21 after BMT were also consistent with a slower return of reticulocytes (WRS, $P=0.012$ ). The activity levels measured at all other times posttransplant were not significantly related to time to engraftment for any of the three lineages.

Influence of febrile episodes on colony-stimulating activities in post-BMT plasma. The observed growth-promoting activities in post-BMT plasma may be related to the release of endotoxin during febrile episodes. We therefore excluded from the analysis 19 samples that were collected during a febrile episode or within $4 \mathrm{~d}$ of defervescence. The activity profiles of this subgroup of 196 samples were indistinguishable from those of all 215 samples depicted in Fig. 1. In addition, 19 patients remained completely afebrile during their post BMT course. Separate assessment of their 111 plasma samples also resulted in similar activity profiles.

Influence of $G v H D$. Based on our previous study (30), GvHD has a major influence on engraftment. We therefore determined whether or not the observed activity profiles for CFU-Meg differed for patients with and without GvHD. As previously observed, patients with GvHD in this study also engrafted slower ( 25 vs. $35 \mathrm{~d}$ : WRS, $P=0.039$ ). Examination for the activity profiles yielded some possible differences. $\mathrm{Pa}$ tients with GvHD appeared to have higher peak activities than patients without GvHD, and patients with GvHD had a tendency to sustain activities longer. However, these differences were not statistically significant at the 0.05 level.

\section{Discussion}

Plasma samples collected from BMT recipients after administration of their respective ablative pretransplant therapy routinely promoted growth of megakaryocyte and granulocytemacrophage colonies in the absence of any additional source of hemopoietic growth factors. The growth-promoting activities usually peaked $7-21 \mathrm{~d}$ after BMT. The return time to pretransplant levels varied considerably from patient to patient. The duration correlated with time to engraftment. $\mathrm{Pa}$ tients that demonstrated growth-promoting activities over prolonged time periods tended to engraft at a slower rate. The growth-promoting activities were observed independent of febrile episodes and documented infections. This information makes it unlikely that growth-promoting activities in postBMT plasma simply reflect the influence of endotoxin.

The neutralization studies using antibodies against GMCSF and IL-3 provided evidence for the view that at least the stimulatory activities for CFU-GM in the six tested plasma samples are consistent with GM-CSF. The simultaneous presence of GM-CSF and EPO may account for the formation of occasional erythroid bursts and multilineage colonies. GMCSF in these plasma samples may also give rise to some megakaryocyte colonies. However, the magnitude of the stimulatory effect for CFU-Meg may suggest the presence of a specific megakaryocyte colony-stimulating activity, since GM-CSF alone only promotes megakaryocyte colony formation by a small subpopulation of available CFU-Meg (26). In addition, the stimulatory activity for CFU-Meg in post-BMT plasma is likely not related to IL-3. Our studies including the neutralization attempts with anti-IL-3 provided little evidence for the release of IL-3.

These initial investigations suggest that hemopoietic growth factors may play a role in the engraftment process. The persistance of growth-promoting activities in the plasma of BMT recipients with delayed engraftment bears resemblance to similar observations in severe aplastic anemia (17) and other cell deficiencies (31-33). Besides cellular defects, one may have to consider deficiencies of factors that interact with earlier pluripotent progenitors such as IL-1 (34) and IL-6 (35). Alternatively, some of the effects may reflect inhibitory mechanisms as previously suggested (36). Future studies are necessary to determine the full spectrum of growth factors, their cellular sources of host and/or donor origin, and mechanisms that are involved in their synthesis and release in relationship to BMT. This information on the endogenous production of growth factors and an understanding of the described growth- 
supporting plasma activities and their fluctuation after BMT may provide further insight into the engraftment process, and may explain mechanisms of delayed engraftment and graft failure. The data will aid in the design and interpretation of clinical trials using human recombinant hemopoietic growth factors in BMT.

\section{Acknowledgments}

The authors thank Drs. Fyles, Cruz, and Scofield and the nursing staff of the transplant unit for their excellent care of patients. Maureen Phelan and Patricia Warrick helped with the preparation of the manuscript.

This study was supported by the Medical Research Council of Canada and the National Institutes of Health.

\section{References}

1. Wong, G. G., J. S. Witek, P. A. Temple, K. M. Wilkens, A. C. Leary, D. P. Luxenberg, S. S. Jones, E. L. Brown, R. M. Kay, E. C. Orr, C. Shoemaker, D. W. Golde, R. J. Kaufman, R. M. Hewick, E. A. Wang, and S. C. Clark. 1985. Human GM-CSF: molecular cloning of the complementary DNA and purification of the natural and recombinant proteins. Science (Wash. DC). 228:810-815.

2. Lee, F., T. Yokota, T. Otsuka, L. Gemmell, N. Larson, J. Luh, K. Arai, and D. Rennick. 1985. Isolation of cDNA for a human granulocyte-macrophage colony-stimulating factor by functional expression in mammalian cells. Proc. Natl. Acad. Sci. USA. 82:4360-4364.

3. Kaushansky, K., P. J. O'Hara, K. Berkner, G. M. Segal, F. S. Hagen, and J. W. Adamson. 1986. Genomic cloning, characterization, and multilineage growth-promoting activity of human granulocytemacrophage colony-stimulating factor. Proc. Natl. Acad. Sci. USA. 83:3101-3105.

4. Nagata, S., M. Tsuchiya, S. Asano, Y. Kaziro, T. Yamazaki, O. Yamamoto, Y. Hirata, N. Kubota, M. Oheda, H. Nomura, and M. Ono. 1986. Molecular cloning and expression of cDNA for human granulocyte colony-stimulating factor. Nature (Lond.). 319:415-418.

5. Souza, L. M., T. C. Boone, J. Gabrilove, P. H. Lai, K. M. Zsebo, D. C. Murdock, V. R. Chazin, J. Bruszewski, H. Lu, K. K. Chen, J. Barendt, E. Platzer, M. A. S. Moore, R. Mertelsmann, and K. Welte. 1986. Recombinant human granulocyte colony-stimulating factor: effects on normal and leukemic myeloid cells. Science (Wash. DC). 232:61-65.

6. Kawasaki, E. S., M. B. Lander, A. M. Wang, J. Van Arsdell, M. K. Warren, M. Y. Coyne, V. L. Schweickart, M. Lee, K. J. Wilson, A. Boosman, E. R. Stanley, P. Ralph, and D. F. Mark. 1985. Molecular cloning of a complementary DNA encoding human macrophage-specific colony-stimulating factor (CSF-1). Science (Wash. DC). 230:291-296.

7. Emerson, S. G., C. A. Sieff, E. A. Wang, G. G. Wong, S. C. Clark, and D. G. Nathan. 1985. Purification of fetal hematopoietic progenitors and demonstration of recombinant multipotential colony-stimulating activity. J. Clin. Invest. 76:1286-1290.

8. Sieff, C. A., S. G. Emerson, R. E. Donahue, D. G. Nathan, E. A. Wang, G. G. Wong, and S. C. Clark. 1985. Human recombinant granulocyte-macrophage colony-stimulating factor: a multilineage hematopoietin. Science (Wash. DC). 230:1171-1173

9. Yang, Y. C., A. B. Ciarletta, P. A. Temple, M. P. Chung, S. Kovacic, J. S. Witek-Giannotti, A. C. Leary, R. Kriz, R. E. Donahue, G. G. Wong, and S. C. Clark. 1986. Human IL-3 (multi-CSF): identification by expression cloning of a novel hematopoietic growth factor related to murine IL-3. Cell. 47:3-10.

10. Lin, F. K., S. Suggs, C. H. Lin, J. K. Browne, R. Smalling, J. C. Egrie, K. K. Chen, G. M. Fox, F. Martin, Z. Stabinsky, S. M. Badrawi, P. H. Lai, and E. Goldwasser. 1985. Cloning and expression of the human erythropoietin gene. Proc. Natl. Acad. Sci. USA. 82:75807584.

11. Eschbach, J. W., J. C. Egrie, M. R. Downing, J. K. Browne, and J. W. Adamson. 1987. Correction of the anemia of end-stage renal disease with recombinant human erythropoietin. Results of a combined phase I and II clinical trial. $N$. Engl. J. Med. 316:73-78.

12. Groopman, J. E., R. T. Mitsuyasu, M. J. DeLeo, D. H. Oette, D. W. Golde. 1987. Effect of recombinant human granulocyte-macrophage colony stimulating factor on myelopoiesis in the acquired immunodeficiency syndrome. N. Engl. J. Med. 317:593-598.

13. Donahue, R. E., E. A. Wang, D. K. Stone, R. Kamen, G. G. Wong, P. K. Sehgal, D. G. Nathan, and S. C. Clark. 1986. Stimulation of haematopoiesis in primates by continuous infusion of recombinant human GM-CSF. Nature (Lond.). 321:872-875.

14. Bonilla, M. A., A. P. Gillio, G. K. Potter, R. J. O'Reilly, T. Boone, L. M. Souza, and K. Welte. 1986. In vivo effects of recombinant human granulocyte colony stimulating factor on chemotherapy induced neutropenia in primates. Blood. 68:160a. (Abstr.)

15. Welte, K., M. A. Bonilla, A. P. Gillio, T. Boone, J. L. Gabrilove, G. Potter, R. J. O'Reilly, and L. M. Souza. 1986. In vivo effects of combined recombinant human G-CSF and GM-CSF on hematopoiesis in primates. Blood. 68:183a. (Abstr.)

16. Gillio, A. P., M. A. Bonilla, R. J. O’Reilly, G. K. Potter, T. Boone, L. M. Souza, and K. Welte. 1986. Effect of recombinant human G-CSF on hematopoietic reconstitution following autologous bone marrow transplantation in primates. Blood. 68:283a. (Abstr.)

17. Solberg, L. A., N. Jamal, and H. A. Messner. 1985. Characterization of human megakaryocytic colony formation in human plasma. J. Cell. Physiol. 124:67-74.

18. de Alarcon, P., and J. A. Schmieder. 1986. Megakaryocyte colony stimulating activity (Mk-CSA) in serum from patients undergoing bone marrow transplantation. In Megakaryocyte Development and Function. R. F. Levine, N. Williams, J. Levin, and B. L. Evatt, editors. Alan R. Liss, Inc., New York. 335-340.

19. Messner, H. A. 1984. Multipotent stem cells in vitro. In Methods in Hematology. D. W. Golde, editor, Churchill Livingstone, New York. 73-86.

20. Curtis, J. E., and H. A. Messner. 1982. Bone marrow transplantation for leukemia and aplastic anemia: management of $\mathrm{ABO}$ incompatibility. Can. Med. Assoc. J. 126:649-655.

21. Fyles, G., H. A. Messner, M. D. Minden, J. E. Curtis, G. Lockwood, and D. Tritchler. 1986. Cyclosporin A in the treatment of acute graft vs host disease. Int. J. Cell Cloning. 4(Suppl. 1):200-202.

22. Messner, H. A., N. Jamal, and C. Izaguirre. 1982. The growth of large megakaryocyte colonies from human bone marrow. J. Cell. Physiol. (Suppl. 1):45-51.

23. Lim, B., N. Jamal, and H. A. Messner. 1984. Flexible association of hemopoietic differentiation programs in multilineage colonies. J. Cell. Physiol. 121:291-297.

24. Aye, M. T., Y. Niho, J. E. Till, and E. A. McCulloch. 1974. Studies of leukemic cell populations in culture. Blood. 44:205-219.

25. Messner, H. A., J. E. Till, and E. A. McCulloch. 1972. Density distributions of marrow cells from mouse and man. Ser. Haematol. 5:22-36.

26. Messner, H. A., K. Yamasaki, N. Jamal, M. D. Minden, Y. C. Yang, G. G. Wong, and S. C. Clark. 1987. Growth of human hemopoietic colonies in response to recombinant gibbon interleukin 3: comparison with human recombinant granulocyte and granulocyte-macrophage colony-stimulating factor. Proc. Natl. Acad. Sci. USA. 84:67656769.

27. Bjorck, L., and G. Kronvall. 1984. Purification and some properties of streptococcal protein G, a novel IgG-binding reagent. J. Immunol. 133:969-974.

28. Glucksberg, H., R. Storb, A. Fefer, C. D. Buckner, P. E. Neiman, R. A. Clift, K. G. Lerner, and E. D. Thomas. 1974. Clinical manifestations of graft-versus-host disease in human recipients of marrow from HL-A-matched sibling donors. Transplantation. 18:295-304. 
29. Randles, R. H., and D. A. Wolfe. 1979. Statistics. In Introduction to the Theory of Statistics. John Wiley \& Sons, New York. 61-112.

30. Messner, H. A., J. E. Curtis, M. D. Minden, D. Tritchler, G. Lockwood, T. Takahashi, J. Lepine, N. Jamal, M. Tweeddale, and U. Wandl. 1987. Clonogenic hemopoetic precursors in bone marrow transplantation. Blood. 70:1425-1432.

31. Mazur, E. M., P. de Alarcon, K. South, and L. Miceli. 1984. Human serum megakaryocyte colony-stimulating activity increases in response to intensive cytotoxic chemotherapy. Exp. Hematol. 12:624-628.

32. Miura, M., C. W. Jackson, and S. A. Lyles. 1984. Increases in circulating megakaryocyte growth-promoting activity in the plasma of rats following whole body irradiation. Blood. 63:1060-1066.
33. Kasper, L. V., and T. M. Seed. 1984. CFU-GM colony enhancing activity in sera of dogs under acute and chronic gamma-irradiation regimens. Acta Haematol. 189-197.

34. Moore, M. A. S., and D. J. Warren. 1987. Synergy of interleukin 1 and granulocyte colony-stimulating factor: in vivo stimulation of stem-cell recovery and hematopoietic regeneration following 5-fluorouracil treatment of mice. Proc. Natl. Acad. Sci. USA. 84:7134-7138.

35. Ikebuchi, K., G. G. Wong, S. C. Clark, J. N. Jhle, Y. Hirai, and M. Ogawa. 1987. Interleukin-6 enhancement of interleukin-3 dependent proliferation of multipotential hemopoietic progenitors. Blood. 70(Suppl. 1):173a. (Abstr.).

36. Li, S., R. Champlin, J. H. Fitchen, and R. P. Gale. 1985. Abnormalities of myeloid progenitor cells after "successful" bone marrow transplantation. J. Clin. Invest. 75:234-241. 